\title{
TRATAMENTO DA ENDOMETRIOSE INDUZIDA CIRURGICAMENTE EM COELHAS PELO LASER DE VAPOR DE COBRE (LVC) ${ }^{1}$
}

\author{
Maurício Paulo Ângelo Mieli² \\ Wanda de Oliveira ${ }^{3}$ \\ Junko Takano Osaka ${ }^{4}$ \\ Marina Penteado Sandoval ${ }^{5}$ \\ Paulo Roberto Bueno Pereira ${ }^{6}$ \\ Erasmo Magalhães de Castro Tolosa ${ }^{7}$
}

\begin{abstract}
Mieli MPA, Oliveira W, Osaka JT, Sandoval MP, Pereira PRB, Tolosa EMC. Tratamento da endometriose induzida cirurgicamente em coelhas pelo laser de vapor de cobre (LVC). Acta Cir Bras [serial online] 2002 Set-Out;17(5). Disponível em URL: http://www.scielo.br/acb.
\end{abstract}

RESUMO - Objetivo: Avaliar o uso do laser de vapor de cobre no tratamento da endometriose cirurgicamente induzida em coelhas. Métodos: 42 coelhas da raça New Zealand foram submetidas à indução experimental da endometriose, de acordo com técnica cirúrgica padronizada. Após 30 dias da indução, um grupo de 29 animais foi submetido ao tratamento pelo laser de vapor de cobre (LVC) em tempos que variaram de 2, 5, 15, 30 e 60 segundos e, outro grupo de 13 coelhas, serviu como controle, tendo-se realizado apenas a laparotomia, sem que fosse realizado algum tratamento. Após 15 dias da segunda laparotomia realizou-se avaliação histopatológica quanto à presença de endometriose, glândulas e estroma. Resultados: O modelo experimental de indução da endometriose foi adequado, com $100 \%$ de eficácia. O tratamento com LVC mostrou-se eficiente, com destruição da doença em 31,00 \% dos casos. Nas coelhas tratadas que ainda tinham a lesão, houve diminuição em 69,85 \% na quantidade de glândulas. A destruição do estroma aconteceu em 41,38\% dos casos, no grupo laser. Conclusão: Os resultados mostraram que o LVC é uma ferramenta adequada para o tratamento da endometriose, como o observado em coelhas.

DESCRITORES - Laser. Coelhas. Endometriose.

\section{INTRODUÇÃO}

O domínio do uso de laser pelos cientistas, construindo instrumentos que permitissem aproveitar suas propriedades, foi de grande importância para a Medicina, levando a um grande número de pesquisas experimentais e ao planejamento terapêutico de várias doenças.
Um dos primeiros trabalhos sobre o uso de laser em seres humanos foi o de Goldman et $\mathrm{al}^{1}$, que demonstraram os efeitos do laser a rubi na pele humana bem como uma das maiores vantagens sobre outros tipos de tratamento: a habilidade de focar energia de alta intensidade em pontos muito pequenos.

Ball $^{2}$ observou que a ação do laser nos tecidos depende basicamente de seu comprimento de onda,

1. Trabalho realizado na Disciplina de Técnica Cirúrgica e Cirurgia Experimental do Departamento de Cirurgia da Faculdade de Medicina da Universidade de São Paulo.

2. Professor Doutor da Disciplina de Técnica Cirúrgica e Cirurgia Experimental do Departamento de Cirurgia da Faculdade de Medicina da Universidade de São Paulo.

3. Professora Doutora do Instituto de Química da Universidade de São Paulo.

4. Bioquímica da Disciplina de Técnica Cirúrgica e Cirurgia Experimental do Departamento de Cirurgia da Faculdade de Medicina da Universidade de São Paulo.

5. Professora Doutora da Disciplina de Patologia do Departamento de Dermatologia da Faculdade de Medicina da Universidade de São Paulo.

6. Professor Associado da Disciplina de Técnica Cirúrgica e Cirurgia Experimental do Departamento de Cirurgia da Faculdade de Medicina da Universidade de São Paulo.

7. Professor Titular da Disciplina de Técnica Cirúrgica e Cirurgia Experimental do Departamento de Cirurgia da Faculdade de Medicina da Universidade de São Paulo. 
intensidade e tempo de exposição. Por outro lado, a capacidade de absorção da energia laser pela água e a cor dos tecidos determinam maior ou menor dano tecidual. A incidência do raio laser pode causar: interação na camada superficial do tecido, dispersão em seu interior, atravessar toda sua estrutura ou ser absorvida.

Mieli et al. ${ }^{3}$ realçaram a preocupação de vários autores, no que diz respeito aos efeitos da radiação óptica nos tecidos quando se analisa os parâmetros físicos e seus efeitos teciduais. Laser que atuam na região ultravioleta do espectro $(100-400 \mathrm{~nm})$, como o laser de argônio e xenônio (excimer), permite incisão e excisão de tecidos por fotoablação e, o "Neodymium-YttriumAluminum-Garnet” (Nd:YAG) possibilita tanto a fotocoagulação como a fotoablação. Em 10.600nm, região do infravermelho do espectro, o laser de $\mathrm{CO}_{2}$ tem sua energia absorvida principalmente pela água das células, tendo sido usado com precisão na ablação de tecidos.

Em comprimento de onda de 400 - 700 nm, região visível do espectro, há muitos efeitos importantes no corpo, freqüientemente mediados pela absorção da radiação pelos pigmentos do tecido (melanina, hemoglobina). Dentre os vários tipos de laser que operam nesta região do espectro, estão incluídos os laser de vapor metálico, sendo o laser vapor de cobre o mais usado dentro desta classe de laser, com comprimentos de onda de 510,5 $\mathrm{nm}$ para a luz verde e de $578,2 \mathrm{~nm}$ para a luz amarela.

Os diferentes tipos de laser, com diferentes comprimentos de ondas foram sendo então, rapidamente aceito como importante ferramenta do arsenal cirúrgico. Em 1993 Sutton $^{4}$ relatou que a mais excitante evolução da terapia em endometriose foi exatamente o surgimento de vários tipos de laser, possibilitando a destruição de implantes ectópicos. $\mathrm{O}$ grande problema surgido foi a dificuldade de se medir a profundidade da destruição: sempre existia o perigo de dano ao intestino e ureter.

Dos vários trabalhos que surgiram utilizando laser no tratamento da endometriose pode-se citar o de Koninckx et al. ${ }^{5}$ que estudaram as complicações e seguimento de pacientes submetidas à excisão de endometriose grave por via endoscópica com laser de $\mathrm{CO}_{2}$ concluindo que a exérese completa por esta via pôde ser realizada em mais de $90 \%$ dos casos; contudo, $6 \%$ das pacientes estudadas necessitaram cirurgia intestinal.

Carter $^{6}$ analisou a performance do uso de laser por fibra óptica de Nd:YAG com extremidade de safira em mulheres submetidas à laparoscopia visando a neurectomia pré-sacral e que tinham diagnóstico de dismenorréia. Verificou ter diminuído a dor após seguimento destas pacientes durante 18 meses. Concluiu ser este procedimento possível, seguro e eficaz, não tendo sido observada nenhuma complicação.
Sutton et al. ${ }^{7}$ verificaram os resultados do tratamento de mulheres que possuíam grandes endometriomas e se submeteram à laparoscopia com intervenção do laser $\mathrm{CO}_{2}$ ou potassium-titanyl-phosphate (KTP). Relataram que houve diminuição da dor e as pacientes que desejavam engravidar, o conseguiram. Concluíram ser o tratamento laparoscópico a laser prático, seguro e eficaz em endometriomas de ovário.

Considerando que a endometriose tem um importante componente vascular envolvido, primeiramente pela presença de fator de crescimento endotelial vascular (VEGF) no líquido peritoneal e, conseqüentemente, à sua neovascularização somando-se ao fato de que um dos comprimentos de onda $(578,5 \mathrm{~nm})$ do laser vapor de cobre está próximo ao pico de absorção da oxihemoglobina, procurou-se neste trabalho avaliar o potencial terapêutico do LVC no tratamento da endometriose, pelos resultados obtidos com a aplicação do LVC na endometriose induzida experimentalmente em coelhas.

\section{MÉTODOS}

O estudo foi realizado em 42 coelhas da raça $\mathrm{New}$ Zealand, nuligestas com peso médio de 3080 gramas, provenientes da Diretoria Técnica de Apoio ao Ensino e Pesquisa da Faculdade de Medicina da Universidade de São Paulo, de acordo com os "Princípios Éticos na Experimentação" da União Protetora dos Animais e Lei 6638 de 08 de Maio de 1979.

O modelo experimental de indução da endometriose seguiu procedimento clássico ${ }^{8}$ conforme resumido a seguir: dissecção do endométrio, descolando-o do miométrio (Figura 1), realizando-se retalho de 5 x 5 × 2 $\mathrm{mm}$ do mesmo (Figura 2). Este retalho foi suturado na face posterior do mesométrio (Figura 3) com fios de mononylon 7-0, de maneira a distar $2 \mathrm{~cm}$ da vagina e 2 $\mathrm{cm}$ do corno uterino direito, através de técnica microcirúrgica, com microscópio Frata, modelo FC-250 e aumento de 12,5 x.

Dividiram-se os animais em 2 grupos, o primeiro grupo composto por 29 coelhas tratadas com LVC e subdividido em 5 subgrupos (Grupo Laser). O segundo grupo de 13 coelhas que serviu como controle (Grupo Controle).

Os animais do grupo laser foram tratados por ocasião da segunda laparotomia e nos do grupo controle, apenas se verificou a presença da endometriose.

O tempo de aplicação da luz (Dt) por ponto e o número de animais de cada subgrupo do Grupo Laser, foram assim divididos: 60 segundos - 8 animais; 30 segundos - 4 animais; 15 segundos - 5 animais; 5 segundos - 6 animais e 2 segundos - 6 animais. 
A luz do LVC sempre esteve em seus dois comprimentos de onda: $510,6 \mathrm{~nm}$ para a luz verde e $578,2 \mathrm{~nm}$ para luz amarela, com uma potência média $\left(\mathrm{P}_{\mathrm{m}}\right)$ de 1,86 $\pm 0,28$ Watt (W), freqüência (f) de $12,6 \times 10^{3}$ Hertz $(\mathrm{Hz})$ e largura de pulso (t) de 30 nanosegundos (ns).

A energia por pulso $\left(\mathrm{E}_{\mathrm{pp}}=\mathrm{P}_{\mathrm{m}} / \mathrm{f}\right)$ utilizada em cada ponto, esteve entre 0,10 a $0,17 \mathrm{~mJ}$, com média de 0,15 $\mathrm{mJ}$. O número de pulsos $(\mathrm{N}=\mathrm{f} \times \mathrm{Dt})$ aplicado em cada ponto esteve entre $25\left(\times 10^{3}\right)$ e $756\left(\times 10^{3}\right)$ pulsos por segundos, com média de $312\left(\times 10^{3}\right)$ pulsos por segundos. O tempo efetivo de exposição $\left(\mathrm{Dt}_{\mathrm{ee}}=\mathrm{N}\right.$ x t) por ponto, variou de 0,001 a $0,023 \mathrm{~s}$, com média de $0,009 \mathrm{~s}$. A energia total $\left(\mathrm{E}=\mathrm{E}_{\mathrm{pp}} \times \mathrm{N}\right.$ Joule $\left.(\mathrm{J})\right)$ aplicada em cada ponto variou de 2,8 a $132 \mathrm{~J}$, com média de 49,7 J.

Incidiram-se os raios do LVC perpendicularmente em cada ponto do tecido, após direcionamento da luz guia do laser Hélio-Neon, de forma que contornasse a lesão de endometriose, tal qual um anel.

Todos os animais foram submetidos à terceira laparotomia, após 15 dias da segunda, e suas áreas do mesométrio, tratadas ou não, foram ressecadas.

\section{RESULTADOS}

A presença da endometriose está apresentada na figura 1. O resultado do tratamento pelo LVC e sua comparação com o grupo controle está demonstrado nas figuras 2 a 4 e gráficos 1 a 3 . A presença de glândulas e estroma, nas figuras 3 e 4, estão representadas pelo critério de cruzes. Os gráficos 1 a 3 representam a destruição da endometriose em relação ao tempo de exposição do LVC.

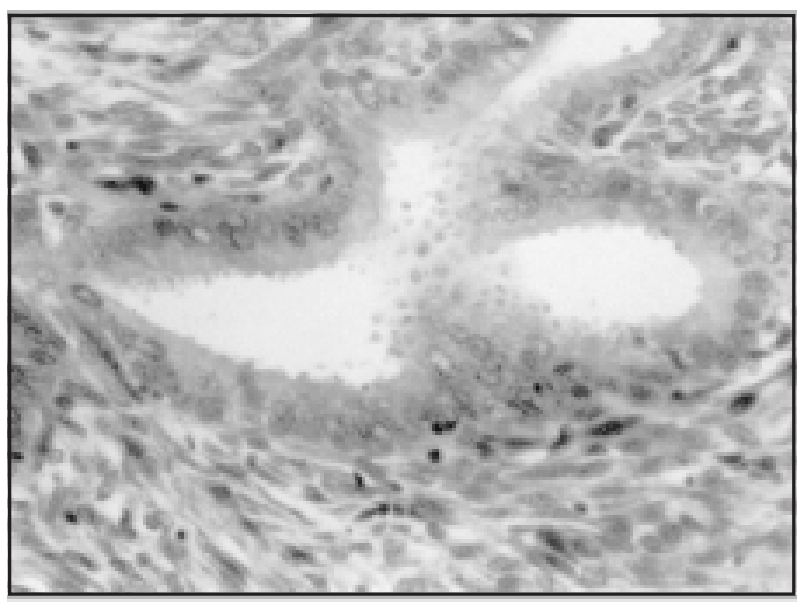

Grupo Laser

FIGURA 1 - Presença das glândulas e estroma (aumento de $40 \mathrm{X})$.

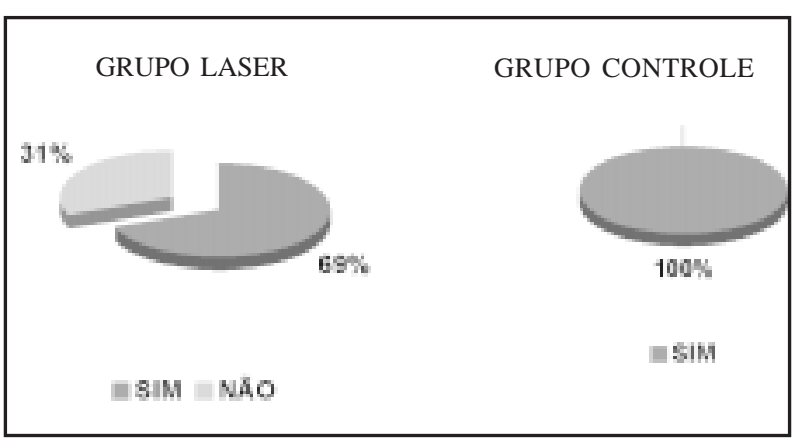

FIGURA 2 - Presença de Endometriose nos Grupos Laser e Controle.

$(\mathrm{SIM}=$ Presença, NÃO = Ausência $)$.

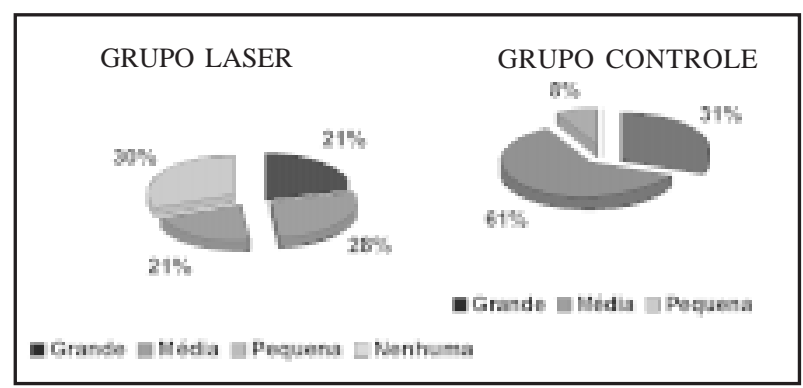

FIGURA 3 - Presença de Glândulas nos Grupos Laser e Controle (Pequena $=+$, Média $=++$, Grande $=+++$ ).

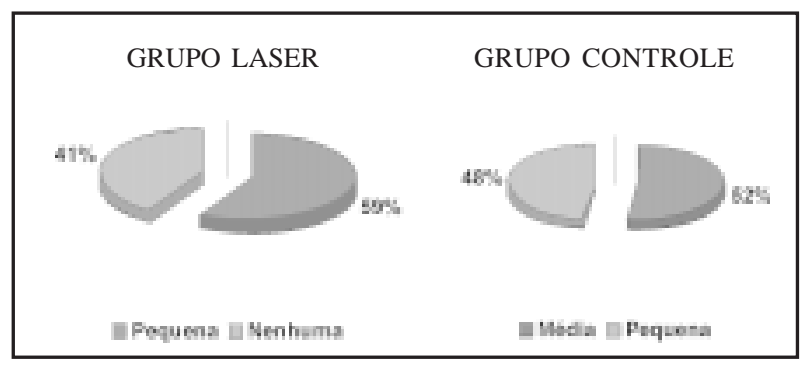

FIGURA 4 - Presença de Estroma nos Grupos Laser e Controle $($ Pequena $=+$, Média $=++)$.

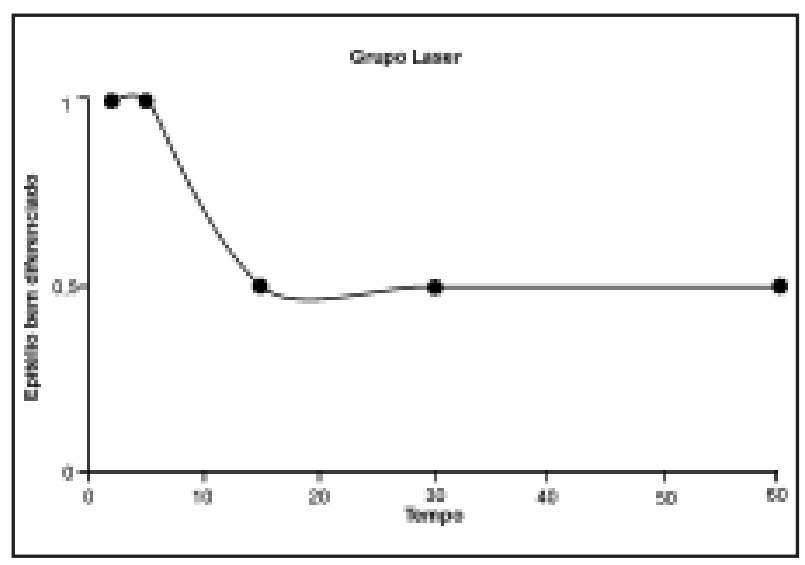

GRÁFICO 1 - Ação do LVC na endometriose. 


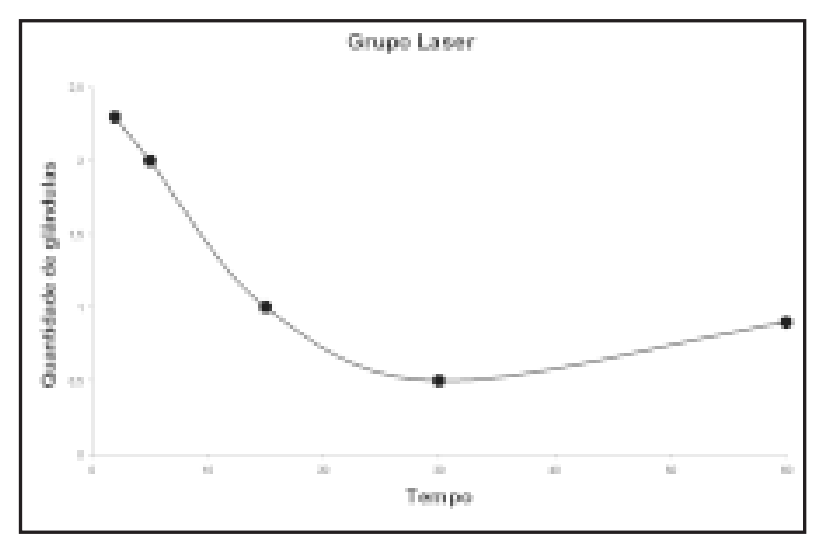

GRÁFICO 2 - Ação do LVC nas glândulas.

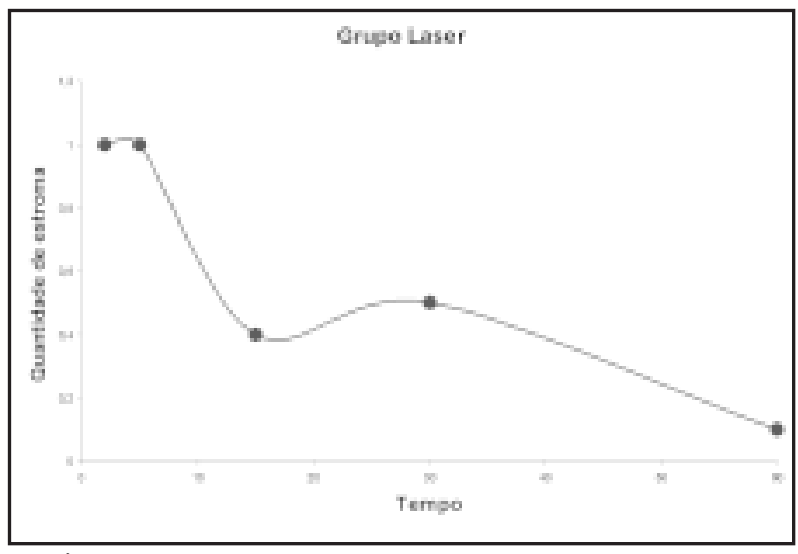

GRÁFICO 3 - Ação do LVC no estroma.

\section{DISCUSSÃO}

Neste trabalho foram utilizados os dois comprimentos de ondas $(510,6$ e 578,2nm) do LVC, apesar de se saber da seletividade tecidual relativa à melhor absorção de um ou outro comprimento de onda. Adotouse este procedimento para que o LVC construído e utilizado estivesse em sua capacidade máxima. Observouse no estudo piloto que, após a utilização do feixe de laser em cada ponto, para a realização do anel, por 60 segundos, houve saída de exsudato seroso que imediatamente se coagulava, formando crosta no local. Surgiu daí a idéia da formação de cinco subgrupos do grupo laser, de 60, 30, 15, 5 e 2 segundos. No último tempo de 2 segundos não mais se verificou saída de exsudato, nem formação de crosta, macroscopicamente.

O conhecimento dos aspectos físicos do LVC (parâmetros apresentados em materiais e métodos) foi oportuno; pois, se o objetivo for aumentar ou diminuir a quantidade de energia total no tratamento da endometriose, pode-se considerar as variáveis com que se trabalha para se atingir tal fim. O fator tempo fez com que se atingisse determinados valores em Joules; contu- do, deve-se lembrar sempre da possibilidade de mudanças na freqüência, ou quiçá, na potência.

O resultado obtido conforme apresentado nas Figuras 1 e 2 mostrou a eficácia do modelo utilizado na indução da endometriose, pois $100 \%$ das coelhas do grupo controle tiveram seus diagnósticos anatomopatológicos comprovados. No grupo tratado com o LVC, apesar de manter-se nódulo de endometriose, após a realização do anel, à aplicação do laser observou-se que $31 \%$ dos casos não apresentaram evidências histológicas de glândulas e/ou estroma. O padrão epitelial de glândulas endometriais bem diferenciadas (Figura 1) foi verificado em todos os casos que tiveram a presença da moléstia.

Houve uma estabilização pela metade na destruição do epitélio glandular bem diferenciado, após o tempo de 15 segundos da aplicação do LVC, conforme gráfico 1. O objetivo foi verificar as alterações histológicas, após o selamento do suprimento sanguiíneo da lesão induzida. Outro fator a ser levado em consideração é o fato de ter-se aplicado o feixe de LVC em um único plano, quando parte da "esfera" do endometrioma se encontrava infiltrada para o interior do tecido mesometrial. Quiçá poder-se-á pesquisar a melhora destes resultados, aumentando-se a energia do LVC, alterandose adequadamente a potência média e/ou a frequiência do LVC utilizado.

Observou-se que a presença glandular (Figuras 1 e 3) se deu em $100 \%$ dos casos no grupo controle e, de uma maneira mais intensa (duas a três cruzes) em 92,31\% das coelhas. No grupo laser a ausência de glândulas ocorreu em 31,03\% dos casos, com queda para $48,28 \%$ dos animais que possuíam maior quantidade de glândulas (duas a três cruzes). O estroma sempre esteve presente no grupo controle (Figuras 1 e 4). A destruição do estroma pelo LVC, foi de $41,38 \%$ dos casos, no grupo laser (Figura 4). A quantidade de estroma neste grupo foi pequena (uma cruz) em 58,62\%, em contraste com $92,31 \%$ do grupo controle e, ausente em quantidades maiores (duas cruzes), contra 7,69\% do grupo controle. Constatou-se, uma tendência a queda progressiva da quantidade de glândulas e estroma, à medida que se aumentou o tempo de aplicação da luz do LVC, conforme os gráficos 2 e 3 .

Considerando-se que não se excisou a lesão da endometriose induzida nas coelhas, visando-se unicamente o bloqueio de sua nutrição vascular ao tratamento pelo LVC, poder-se-ia, tendo-se em vista os resultados obtidos neste estudo, utilizar esta estratégia (bloqueio da nutrição vascular) no tratamento das formas graves de endometriose em seres humanos, como as classificadas por Nisolle e Donnez ${ }^{9}$ do septo retovaginal, de difícil abordagem cirúrgica, minimizando-se, portanto, os riscos de lesões iatrogênicas das estruturas vizinhas. 


\section{CONCLUSÃO}

Pelos resultados aqui obtidos no tratamento da endometriose induzida experimentalmente em coelhas da raça New Zeland, pode-se considerar o LVC uma ferramenta adequada para uso no tratamento de endometriose.

\section{AGRADECIMENTOS}

Ao Centro Tecnológico da Marinha em São Paulo (CTMSP).

\section{REFERÊNCIAS}

1. Goldman L, Blaney DJ, Kindel, DL. Pathology of the effect of laser beam on the skin. Nature 1963;197:912-4.

2. Ball KA. Lasers: the perioperative challenge. St. Louis: MosbyYear Book, Inc.; 1995.
3. Mieli MPA, Castelfranchi PL, Matsunaga P, Vilaça PRJr, Pereira PRB. Laser em ginecologia. Femina 1999;27:719-22.

4. Sutton CJ. Laser treatment of endometriosis. Practitioner $1993 ; 237: 601-7$

5. Koninckx PR, Timmermans B, Meuleman C, Penninckx F. Complication of CO2-laser endoscopic excision of deep endometriosis. Hum Reprod 1996;11:2263-8.

6. Carter JE. Laparoscopic presacral neurectomy utilizing contacttip Nd:YAG laser. Keio J Méd 1996;45:332-5.

7. Sutton CJ, Ewen SP, Jacobs SA, Whitelaw NL. Laser laparoscopic surgery in the treatment of ovarian endometriomas. J Am Assoc Gynecol Laparosc 1997;4:319-23.

8. Mieli MPA, Pereira PRB, Oliveira W, Osaka JT, Sandoval, MP, Tolosa EMC. Indução da endometriose em coelhas: um modelo experimental. Enviado para publicação na Revista Brasileira de Ginecologia e Obstetrícia (RBGO) em Setembro de 2001.

9. Nisolle M, Donnez J. Peritonial endometriosis, ovarian endometriosis, and adenomyotic nodules of the rectovaginal septum are three different entities. Fertil Steril 1997; 68:585-96

Mieli MPA, Oliveira W, Osaka JT, Sandoval MP, Pereira PR, Tolosa EMC. The treatment of endometriosis induced surgically in rabbits using copper vapor laser (CVL). Acta Cir Bras [serial online] 2002 Sept-Oct;17(5). Available from URL: http://www.scielo.br/acb.

ABSTRACT - Objective: To evaluate the use of copper vapor laser in treatment of the surgically induced endometriosis in rabbits. Methods: 42 New Zeland rabbits were submitted to the experimental induction of the endometriosis, in agreement with standardized surgical technique. After 30 days of the induction, a group of 29 animals was submitted to the treatment by copper vapor laser (CVL) in times that varied of 2, 5, 15, 30 and 60 seconds and, other group of 13 rabbits served as control without any treatment. After 15 days of the second laparotomy, histopatologic evaluations have done with relationship to the endometriosis presence, glands and estroma. Results: The experimental model of endometriosis induction was adequated, with $100 \%$ of effectiveness. The treatment with LVC was shown efficient, with destruction of the disease in $31,00 \%$ of the cases. In the rabbits that still had the lesion, there was decrease in $69,85 \%$ of glands amount. The destruction of the stroma ocurred in $41,38 \%$ of the cases, in the group laser. Conclusion: The results showed that the CVL is a adequated tool for the endometriosis treatment as observed in rabbits.

KEY WORDS - Laser. Rabbits. Endometriosis.

\section{Endereço para correspondência:}

Conflito de interesse: nenhum Fonte de financiamento: nenhuma

Maurício Paulo Angelo Mieli

Rua Comendador Miguel Calfat, 128/1401

04537-080 São Paulo - SP

Tel.: (11)3487-3955 Fax: (11)3045-2420

mieli@usp.br

Data do recebimento: 25/06/2002

Data da revisão: 12/07/2002

Data da aprovação: 02/08/2002 